\title{
Genomic characterization of Pseudomonas bacteriophage AN14
}

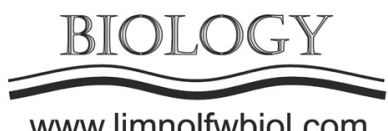

www.limnolfwbiol.com

\author{
Evseev P.V. ${ }^{1}$, Gorshkova A.S. ${ }^{2}$, Sykilinda N.N. ${ }^{1}$, Drucker V.V. ${ }^{2}$, Miroshnikov K.A. ${ }^{1 *}$ \\ ${ }^{1}$ Shemyakin-Ovchinnikov Institute of Bioorganic Chemistry, Russian Academy of Sciences, Miklukho-Maklaya Str., 16/10, Moscow, \\ 117997, Russia \\ ${ }^{2}$ Limnological Institute, Siberian Branch of the Russian Academy of Sciences, Ulan-Batorskaya Str., 3, Irkutsk, 664033, Russia
}

\begin{abstract}
Siphoviruses with a prolate capsid infecting Pseudomonas sp. were isolated from environmental sources through the world, and were recently defined as a separate taxonomic genus Yuavirus. Besides a distinguishable morphology, the hallmarks of the genus are heavily modified genomic DNA and a lytic infection cycle while putative lysogeny genes are proposed in the genomes. Bacteriophage AN14 was isolated from Lake Baikal ecosystem and was assigned as a member of Yuavirus. We report the biological and morphological features if this phage, as well as the comprehensive re-annotation of its genome. Modern bioinformatics analysis resulted in the refinement of the taxonomic attributation of the YuA-like phages and highlighting the specific genomic and proteomic features typical for Yuavirus phages including AN14.
\end{abstract}

Keywords: Bacteriophage, Pseudomonas, genomics, phylogeny, taxonomy

\section{Introduction}

Bacteriophage AN14 infecting Pseudomonas was discovered in the aquatic ecosystem of Lake Baikal in 2010. According to the current nomenclature justified by the International Committee for Virus (ICTV) in 2020, phage AN14 belongs to the genus Yuavirus of family Siphoviridae. ICTV database lists six previously reported phages as members of Yuavirus- Alphaproteobacteria virus $\varphi J L 001$, and Pseudomonas viruses LKO4, M6, MP1412, PAE1 and YuA. Moreover, the NCBI database and taxonomic browser contains information on another 12 phages presumably belonging to the genus Yuavirus. Extreme diversity of the bacterial hosts of this phage group comprising alpha-, beta- and gammaproteobacteria raises questions on the correction and possible revision of the current taxonomic classification.

The presented work reports the parameters of the AN14 infection cycle and morphology. We have re-annotated the AN14 genome taking into account the recent data on structures and functions of phage proteins. Genomic and phylogenetic research have refined the taxonomic position of phages belonging to genus Yuavirus.

\section{Results and discussion}

The genome of AN14 contains 86 putative ORFs, putative functions of 58 proteins can be predicted, and 28 ORFs are assigned as hypothetical proteins. There are no tRNA genes found in the genome. All genes are oriented in the same direction. $\mathrm{G}+\mathrm{C}$ content of the AN14 genome is $64.5 \%$, evenly distributed through the genome. Yuavirus phages infecting Pseudomonas have the $\mathrm{G}+\mathrm{C}$ value most close to the average $\mathrm{G}+\mathrm{C}(65 \%)$ of the bacterial host.

The genes of AN14 genome are clustered in three blocks - the first one encodes structural and lysis proteins, the second one is responsible for nucleotide metabolism and modification proteins, and the third block encodes replication, transcription and other proteins. The genes of structural proteins comprise about one third of the genome and are well conserved in the representatives of the genus. The functions of most of these genes can be predicted using BLAST and HMM-HMM pipelines.

The block for terminal lysis of the host cell in Pseudomonas Yuavirus phages contains genes encoding peptidoglycan-lysing enzyme, which has been proposed to function as murein transglycosylase $\mathrm{F}$, and the holin located immediately downstream. One of the noticeable features of AN14 predicted proteome is a significant number of proteins involved in nucleotide modification and repair. At least 8 genes for such proteins can be found, and their homologs are present in other Yuavirus genomes. A large set of potential nucleotide modification proteins correlates with the experimentally found high content of modified bases in the genomes of phages YuA (Ceyssens et al., 2008). Another interesting feature of

*Corresponding author.

E-mail address: kmi@ibch.ru (K.A. Miroshnikov)

(C) Author(s) 2020. This work is distributed under the Creative Commons Attribution 4.0 License. 
AN14 genome is the presence an anti-restriction system (Spoerel et al., 1979), including antirestriction protein (gp49) and predicted exonuclease inhibitor protein (gp03). The previous annotation of AN14 genome and the annotations of the genomes of YuA-like phages genomes have stated a presence of an integrase-like protein (gp27), but we propose that this protein is most likely a DNA primase.

In order to construct a consistent taxonomy and phylogenetic positioning of phage AN14, we performed a BLAST search using the terminase large subunit protein sequences and GenBank phage database, and constructed a list of phages belonging to the taxa, representatives of which were found with $\mathrm{E}$-value $<10^{-8}$. The maximum likelihood (ML) phylogeny of terminase large subunit protein sequence clearly showed the affiliation of AN14 with the Yuavirus clade. The tree groups 16 Pseudomonas phages into a distinct clade and points to Vojvodinavirus as the genus closest evolutionally. Contrary to the current ICTV classification, Alphaprotebacteria phage 甲JL001 (AY576273) (Lohr et al., 2005)469 bp with an overall G $+C$ content of $62 \%$. The genome has 91 predicted open reading frames (ORFs seems to belong to a group comparatively distant from Yuavirus, and Bordetella virus LK3 (KX961385) belongs to the genus Vojvodinavirus. These conclusions are in the agreement with proteome clustering, ANI calculations, genome sequence comparison among 10 AN14-related phage genomes, and ML phylogeny based on 39 core proteins concatenated sequences.

The terminase phylogeny points to Pseudomonas phages of the Abijanvirus genus as the next closest group to Vojvodinavirus. More distant relatives also represent the phages of gram-negative bacteria, mostly Pseudomonas spp. Phylogenetic analysis suggests that Alphaprotebacteria phage $\varphi \mathrm{JL001}$ terminase have diverged from the common clade comprising Yuavirus and related groups even earlier than the divergence to genera Stenhofvirus, Pamexvirus, Abijanvirus and Vojvodinavirus occured.
Previously, it was shown experimentally that Pseudomonas phage YuA and Bordetella phages, closely related to AN14, infect their hosts through a lytic cycle only. The main rationale to state a possible temperate lifestyle of Yuavirus phages is the presence of the putative conserved integrase gene ( $g 27$ in case of AN14) and the repressor ( $g 26)$ in the genome. However, the homologs of AN14 gp27, annotated in $\varphi J L 001$, YuA, M6 and other related phages as integrases, may perform a different function not related to lysogeny. Alternatively, it may be attributed as DNA primase/ polymerase belonging to AEP-primases. Undoubtedly, this hypothesis requires a future experimental proof. However, it gives a reasonable explanation for the long-disputed contradiction between the presence of predicted lysogeny genetic apparatus, and lysogeny never observed experimentally for Yuavirus phages.

\section{Aknowlegements}

This research was funded by the national government and was carried out within the framework of State Tasks No. 0345-2019-0003 (AAAA-A16116122110061-6) "Microbial and viral communities in biofilms of freshwater ecosystems..."

\section{References}

Ceyssens P.J., Mesyanzhinov V., Sykilinda N. et al. 2008. The genome and structural proteome of $\mathrm{YuA}$, a new Pseudomonas aeruginosa phage resembling M6. Journal of Bacteriology 190: 1429-1435. DOI: 10.1128/JB.01441-07

Lohr J.E., Chen F., Hill R.T. 2005. Genomic analysis of bacteriophage $\varphi \mathrm{JL} 001$ : insights into its interaction with a sponge-associated alpha-proteobacterium. Applied and Environmental Microbiology 71: 1598-1609. DOI: 10.1128/ AEM.71.3.1598-1609.2005

Spoerel N., Herrlich P., Bickle T.A. 1979. A novel bacteriophage defence mechanism: the anti-restriction protein. Nature 278: 30-34. DOI: $10.1038 / 278030 a 0$ 